\title{
Adhesion effects on contact opening dynamics in micromachined switches
}

\author{
Brian D. Jensen, Kuangwei Huang, Linda L.-W. Chow, and Katsuo Kurabayashia \\ Department of Mechanical Engineering, University of Michigan, Ann Arbor, Michigan 48109
}

(Received 24 November 2004; accepted 15 March 2005; published online 16 May 2005)

\begin{abstract}
We propose a technique to measure the opening time for micromachined switches and present substantial experimental data for switches with gold-gold contacts. The data demonstrate that contact opening time increases dramatically as apparent contact area increases or as pull-apart force or contact resistance decreases. A model of opening time is also presented with model parameters that fit the experimental data. Moreover, we show that transient mechanical vibrations can play an important role in reducing switch opening time. () 2005 American Institute of Physics.
\end{abstract}

[DOI: $10.1063 / 1.1901837]$

\section{INTRODUCTION}

Micromechanical switches outperform their solid-state counterparts by almost every measure. Their low power consumption, high isolation, low insertion loss, and linearity make them attractive components in many applications, especially radio-frequency circuits. ${ }^{1}$ However, they switch much more slowly than solid-state devices, and their lifetime and reliability are not as great. Improvements in these areas require a more thorough understanding of adhesion at micromechanical contacts, which affects both switching dynamics and reliability (stiction).

Much of the previous work on microdevice adhesion has focused on the second failure mechanism, stiction. Many researchers have approached adhesion from a fracture mechanics perspective, developing detailed models and making measurements based on the adhesion of cantilevers. ${ }^{2-5}$ Other adhesion models have relied on Hertzian contact mechanics. ${ }^{6}$ Moreover, virtually all published measurements of microscale adhesion have used quasistatic observation of stuck parts. ${ }^{4,7}$ While this approach has proven very powerful, it does not address the first failure mode noted above-no information is gained about the time required for the surfaces to pull apart. In fact, switch opening time is often more critical than stiction failure, since a nonsticking switch that requires excessive time to open is not generally useful. In addition, measurements that rely on surface sticking are, in most cases, destructive. Nondestructive adhesion measurements allow multiple tests per device, yielding much more data for the detection of trends and for model fitting.

This paper reports measurements of adhesion effects in micromachined gold-gold contact switches. Using an oscilloscope, we record the voltage on the switch contact as it opens, allowing an accurate measurement of the opening time. We present experimental data showing that opening time increases with larger apparent contact area, reduced contact resistance, and smaller pull-apart force. We also present a model describing the switch opening time and determine model parameters to fit the data. Finally, we show

${ }^{a)}$ FAX: (734) 647-3170; electronic mail: katsuo@umich.edu that mechanical vibrations of the switch can significantly reduce the opening time. The model and experiments provide valuable information for switch design.

\section{EXPERIMENTAL SETUP}

Figure 1 shows a sample switch used in these tests. The overlaid schematic shows the probe placement used to measure resistance using the four-point probe technique. The switches were micromachined from sputtered gold, with the underlying actuation and contact electrodes also of sputtered gold. The contact location and size were controlled using a dimple on the underside of a beam. The area of the bottom of the dimple is called the apparent contact area since it represents the area in apparent contact. This apparent contact area was controlled lithographically by fabricating switches with a wide range of dimple sizes. All of the switches consisted of fixed-fixed beams with a width of $100 \mu \mathrm{m}$, thickness of $3.1 \mu \mathrm{m}$, and gap under the beam of $1.54 \mu \mathrm{m}$. The dimple height was $1.18 \mu \mathrm{m}$, leaving a distance of $0.36 \mu \mathrm{m}$ to travel before making contact. The length of the switches tested here was either 300 or $400 \mu \mathrm{m}$. The switches were created using surface micromachining, with photoresist as the sacrificial layer and sputtered gold as the structural layer. Fabrication details are given in Ref. 8.

The switches were tested in a vacuum chamber kept at 5-8 mTorr, which is sufficient to reduce the water vapor in the chamber but not to create ultraclean surfaces. Hence, it is likely that a thin hydrocarbon film remains on the surface of

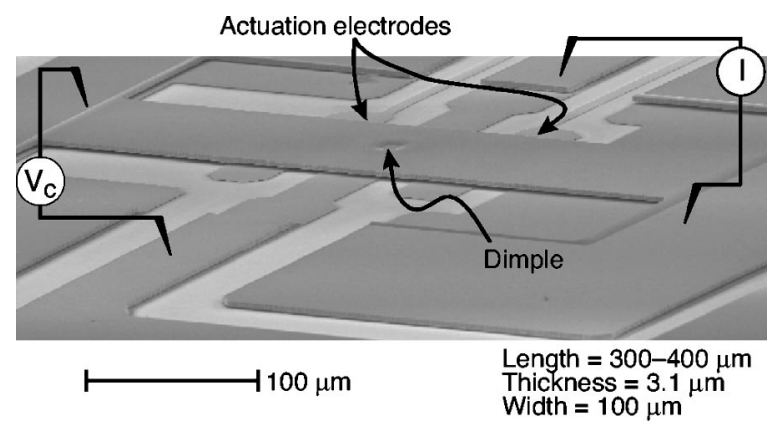

FIG. 1. Sample switch showing the probe placement in a four-point probe technique. 


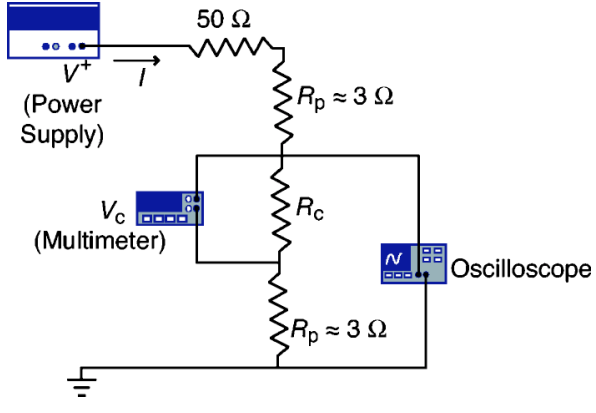

FIG. 2. Measurement circuit schematic. $R_{c}$ is the contact resistance of the switch, $V_{c}$ is the voltage on the contact resistance, and $R_{p}$ is the probe contact resistance.

the gold electrodes. ${ }^{9}$ Testing revealed that while the switches stuck down easily in air, they were unlikely to remain stuck while in the vacuum chamber. Hence, the chamber was successful in reducing adhesion, most likely due to the minimization of humidity effects. The switches were actuated using a function generator attached to a voltage amplifier. Using a square pulse as input, the actuation voltage switched between 0 and 55-128 $\mathrm{V}$ (the actuation voltage range used in these experiments) in about $1.5 \mu \mathrm{s}$, which is much less than the times required for contact opening.

Using the circuit illustrated in Fig. 2, the contact resistance was measured using digital multimeters with the fourpoint probe technique, with a DC power supply providing the measurement current at a constant voltage of $0.2 \mathrm{~V}$. We found that experiments performed at significantly higher open-circuit voltage tended to show considerable variability and instability in the contact resistance. A voltage of $0.2 \mathrm{~V}$ is high enough to measure easily using the multimeters and oscilloscope, but low enough to avoid these problems. A $50-\Omega$ resistor was placed in series with the switch to create a voltage divider circuit, thus enhancing the difference between the on-state and off-state contact voltages. There is also a probe contact resistance $R_{p}$ of about $3 \Omega$ at the contact between the probes and the gold pads. In the schematic, $R_{c}$ represents the contact resistance and $V_{c}$ is the voltage drop at the contact.

The contact voltage was simultaneously measured using an oscilloscope. Figure 3 shows a sample oscilloscope trace during the opening of a switch with dimple area $A_{a}$ $=27.3 \mu \mathrm{m}^{2}$. The plot shows a reduction in the actuation voltage from about 118 to $0 \mathrm{~V}$ (the time when the actuation

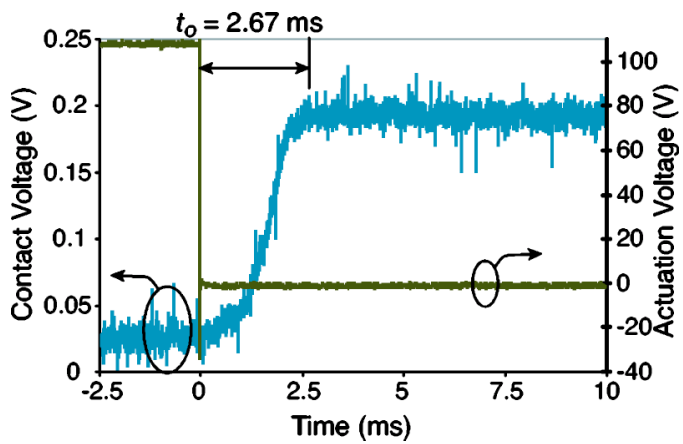

FIG. 3. (Color online) Oscilloscope traces of actuation and contact voltage for a switch with $A_{a}=27.3 \mu \mathrm{m}^{2}$. The contact opening time is marked. voltage is turned off is arbitrarily labeled as $0 \mathrm{~ms}$ ). When the actuation voltage drops to 0 , the force holding the contacts together is removed. However, adhesion in the contact prevents immediate switch opening. Instead, the contact voltage shows a gradual increase from about $0.02 \mathrm{~V}$ to the opencircuit voltage of $0.2 \mathrm{~V}$. The contact opening time $t_{o}$ is measured from the time the actuation voltage is turned off to the time when the contact voltage reaches $0.195 \mathrm{~V}$, or $97.5 \%$ of the open-circuit voltage. Note that the voltage measured by the oscilloscope includes the voltage on one of the probe contacts because our oscilloscope can only measure voltage relative to a common ground. However, since the resistance $R_{p}$ is constant, it has no effect on the measurement of contact opening time. We also estimate the resistance-capacitance time constant of the switch to be less than $1 \mathrm{~ns}$, even for very high contact resistances (a few thousand Ohms). This time constant is more than 1000 times smaller than the fastest opening times we measured, indicating that electrical transients do not affect the measurements.

\section{RESULTS}

Using the technique described above, switches were tested to determine their opening times for a wide variety of conditions. The switches varied in their apparent contact area $A_{a}$ and pull-apart force $F_{\mathrm{pa}}$. As previously stated, the switch contacts had different apparent contact areas because the dimples were designed in different sizes. The apparent contact area for each dimple size was measured by examining the underlying sacrificial layer of photoresist under a microscope before sputtering gold. The pull-apart force $F_{\mathrm{pa}}$ was varied by changing the length of the switch beam. The switches tested here had a beam length of either 300 or $400 \mu \mathrm{m}$. The pull-apart force was extracted from a beam deflection model. ${ }^{10}$ In addition, the switches were tested at varying contact forces (the force acting on the contact while the switch is held down) by changing the actuation voltage.

When two rough surfaces are brought together, they achieve actual contact at a finite number of asperities. Constriction of current flowing through the real contact spots creates an effective resistance known as the contact resistance. ${ }^{11}$ In cases where an insulating film covers a metal surface, current flows only through spots where the film has been deformed to allow metal-to-metal contact. Since micromachined gold surfaces seem to normally have a thin insulating layer, this effect becomes very important. ${ }^{9}$ For a circular metal-to-metal contact spot, the contact resistance is ${ }^{12}$

$$
R_{c}=\frac{4 \rho_{e} \lambda}{3 \pi a^{2}}+\left[\frac{1+0.83(\lambda / a)}{1+1.33(\lambda / a)}\right] \frac{\rho_{e}}{2 a},
$$

where $R_{c}$ is the contact resistance, $\rho_{e}$ is the electrical resistivity in the metal (assumed to be the same for both surfaces), $\lambda$ is the mean free path of an electron, and $a$ is the radius of the contact spot.

This equation gives the resistance for a single spot; however, a given contact is expected to have multiple contact spots. Nevertheless, because the largest contact spot contributes most to the contact conductance, the contributions of the smaller spots may often be ignored. This may be shown us- 
TABLE I. Characteristics of the seven switches tested here, showing apparent contact area $A_{a}$, pull-apart force $F_{\mathrm{pa}}$, and beam length $l$.

\begin{tabular}{cccc}
\hline \hline Switch No. & $\begin{array}{c}A_{a} \\
\left(\mu \mathrm{m}^{2}\right)\end{array}$ & $\begin{array}{c}F_{\mathrm{pa}} \\
(\mu \mathrm{N})\end{array}$ & $\begin{array}{c}l \\
(\mu \mathrm{m})\end{array}$ \\
\hline 1 & 26.7 & 76.6 & 300 \\
2 & 27.3 & 76.6 & 300 \\
3 & 29.6 & 76.6 & 300 \\
4 & 29.6 & 76.6 & 300 \\
5 & 29.6 & 54.4 & 400 \\
6 & 169 & 76.6 & 300 \\
7 & 314 & 76.6 & 300 \\
\hline \hline
\end{tabular}

ing fractal modeling of the contact surface. For a given fractal surface, the total real contact area is related to the area of the largest contact spot through the relation ${ }^{13}$

$$
A_{T}=\frac{D-1}{3-D} A_{L},
$$

where $A_{T}$ is the total contact area, $D$ is the fractal dimension (a parameter between 2 and 3), and $A_{L}$ is the area of the largest contact spot. Hence, if $D$ is close to $2, A_{T} \approx A_{L}$. By comparing fractal surfaces to an atomic force microscope scan of the sputtered gold used in our experiments, we determined that $D$ for our surface is approximately 2.01 , so that $A_{T}=1.02 A_{L}$. Therefore, in this work, we ignore the effects of smaller contact spots by assuming a single-asperity contact.

Previous work has demonstrated that mechanical cycling causes an increase in contact resistance, while raising the contact voltage causes a decrease in contact resistance. ${ }^{8} \mathrm{We}$ used this behavior to make measurements over a wide range of measured contact resistances. A total of 984 measurements were made on seven switches. The characteristics of each switch are given in Table I. The apparent contact area varied from 26.7 to $314 \mu \mathrm{m}^{2}$, and pull-apart force was either 54.4 or $76.6 \mu \mathrm{N}$, depending on the length of the switch beam. The results for switches 3 and 4 are reported together since their characteristics were the same $\left(A_{a}\right.$ of $29.6 \mu \mathrm{m}^{2}$ and $F_{\mathrm{pa}}$ of $76.6 \mu \mathrm{N})$. Moreover, no difference could be discerned between the data taken from these two switches. The data is summarized in Fig. 4, showing the dependence of opening time on apparent contact area, real contact radius, and pullapart force. The measured contact resistance corresponding to the real contact radius is also shown, as calculated using Eq. (1). For this calculation, we used a mean free path of $38 \mathrm{~nm}$ (from Ref. 14) and a gold resistivity of $3.6 \times 10^{-8}$ $\Omega-m$. We measured the resistivity using on-chip van der Pauw structures. Note that throughout this paper, the real contact radius is defined as the effective contact radius, as calculated using Eq. (1), at the beginning of the opening process. In other words, it is the contact radius corresponding to the contact resistance in the switch before it opens.

The figure indicates that contact opening time increases dramatically with increasing apparent contact area and real contact radius, and decreases with increasing pull-apart force. This implies that contacts with larger apparent contact area have more adhesion, regardless of the real contact size. This could be due to enhanced van der Waals interactions between the surfaces, or to hydrogen bonds or even molecular bonds which form between the surface films on the surfaces when they are pressed together. Moreover, contacts with smaller contact resistance have a larger real contact area, which also leads to more adhesion. Since smaller contact resistance implies that more gold bonds are created at the contact spot, we expect that breaking these bonds would require more energy and time as compared to a smaller contact spot (with larger contact resistance). Finally, contacts pulled apart with larger force tend to open more quickly, as expected.

Figure 4 shows considerably more variability for the two switches with the largest apparent contact area. Much of this variability can be accounted for by considering the contact

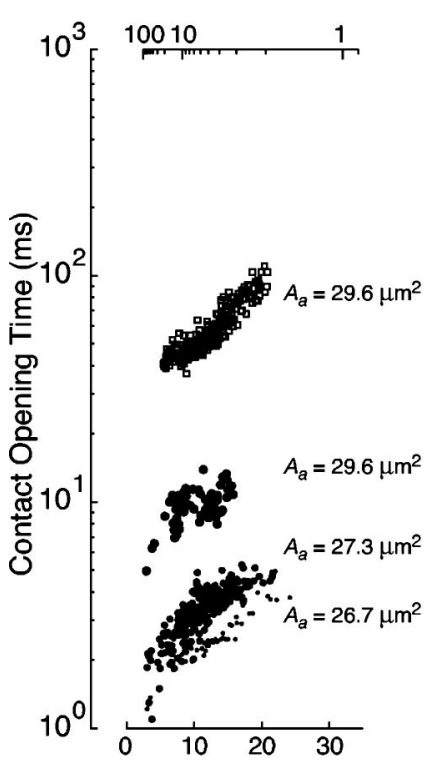

$26.7-29.6$

\section{Contact Resistance $(\Omega)$}
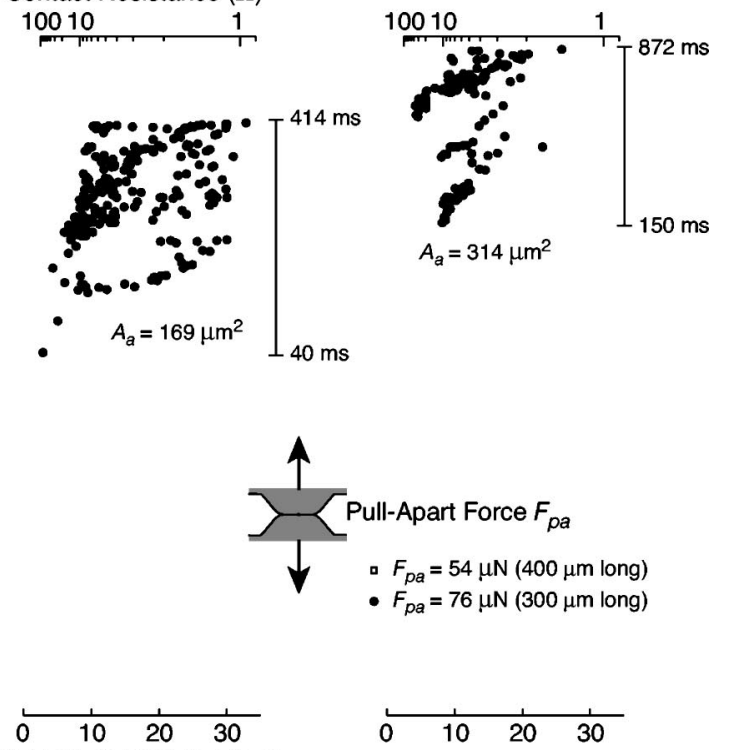

Real Contact Radius (nm)

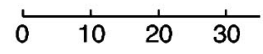

FIG. 4. Switch opening time data for all switches showing dependence on real contact radius, apparent contact area, and pull-apart force.

Apparent Contact Area $\mathrm{A}_{\mathrm{a}}\left(\mu \mathrm{m}^{2}\right)$ 


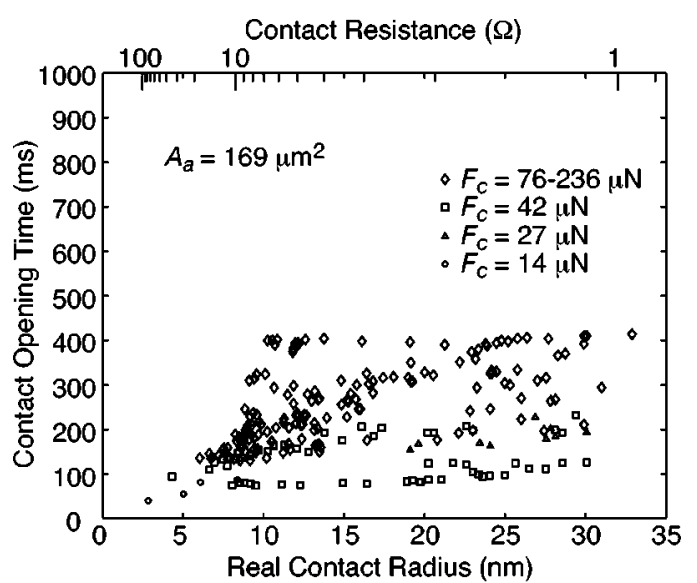

(a)

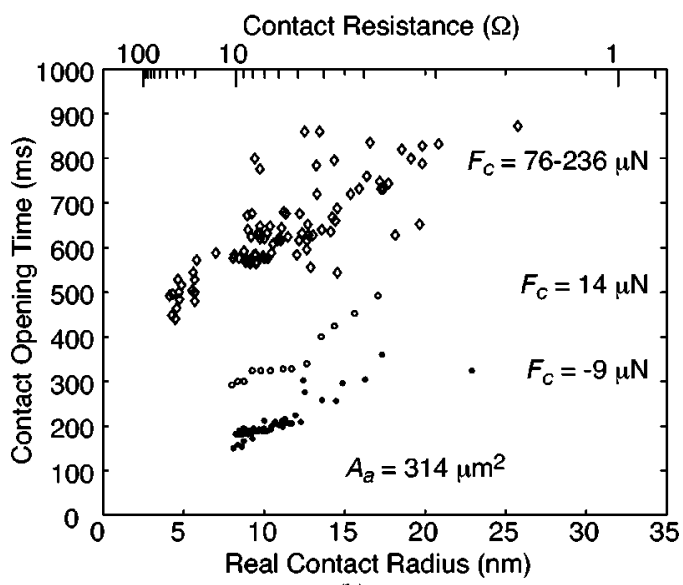

(b)

FIG. 5. Switch opening time for (a) $A_{a}=169 \mu \mathrm{m}^{2}$ and (b) $A_{a}=314 \mu \mathrm{m}^{2}$ with the contact force indicated by varying symbols.

force. The contact force is the force acting to push the contact together before contact opening begins; that is, it is the force which creates the contact. Contact force can be varied for the switches used here by changing the actuation voltage acting to close the switch. As already mentioned, the data in Fig. 4 were taken for contacts formed using a variety of contact forces. Figure 5 shows the data for the two switches with the largest apparent contact area, with the contact force represented by varying symbols. For this data, the contact force was extracted from measurements of actuation voltage using a mechanical model of the switch. ${ }^{10}$ The resulting contact force is, of course, the force neglecting adhesion, so that even slightly negative reported contact forces still show low resistance because adhesive forces maintain contact. For both switches, the smallest contact forces give the lowest contact opening times, with increasing time as force rises until the effect disappears above about $76 \mu \mathrm{N}$. As the modeling will suggest, we believe this effect is due to the reduced pressure pushing the surfaces together, resulting in fewer interactions between the surfaces.

\section{THEORY AND MODEL}

Figure 6 illustrates the contact of two metal surfaces covered with a thin insulating layer, as appears to be the case for our switches. It shows contact at asperities where the thin

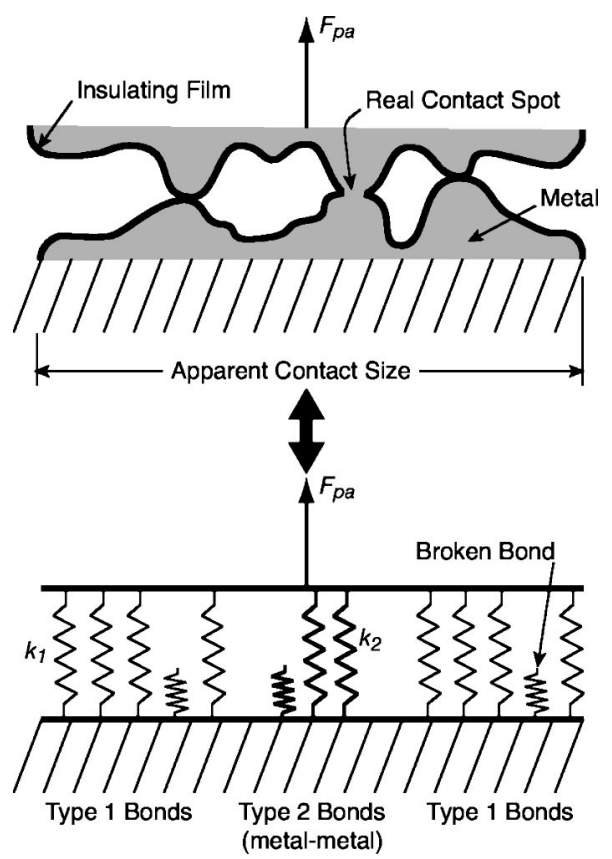

FIG. 6. Illustration of contact between two metal surfaces coated with a thin insulating layer. A model to describe the opening of the contact is also shown.

film is not punctured as well as metal-to-metal contact at an asperity where the insulating film has broken. When the surfaces are pulled apart with a force $F_{\text {pa }}$, the lower illustration shows how the contact opening may be modeled. This model is based on a model proposed to describe adhesion between biological cells. ${ }^{15}$ Since the model represents all bonds generically as springs, it is easily generalized to our case. The model recognizes two types of bonds in the contact area. Type 1 bonds represent van der Waals bonds, hydrogen bonds, or any other types of bonds found within the apparent contact area but not at the real contact spot. These bonds account for the increased adhesion observed when the apparent contact area increases. Type 2 bonds represent metal-tometal bonds formed at the real contact. If the bonds are treated as linear springs, then we may write

$$
F_{\mathrm{pa}}=K_{T} x_{T}=\left(N_{1} K_{1}+N_{2} K_{2}\right) x_{T},
$$

where $K_{T}$ is the total spring constant, $x_{T}$ is the spring displacement, $N_{1}$ and $N_{2}$ are the total numbers of type 1 and type 2 bonds, and $K_{1}$ and $K_{2}$ are the spring constants for each type of bond. The force acting on a single bond of types 1 and 2 is then

$$
\begin{aligned}
& F_{1}=K_{1} x_{T}=\frac{K_{1} F_{\mathrm{pa}}}{N_{1} K_{1}+N_{2} K_{2}}, \\
& F_{2}=K_{2} x_{T}=\frac{K_{2} F_{\mathrm{pa}}}{N_{1} K_{1}+N_{2} K_{2}} .
\end{aligned}
$$

Opening of the contact requires bond dissociation. Chemical kinetics, using Boltzmann statistics, suggests the bond dissociation rate, ${ }^{16,17}$

$$
k_{1}(t)=k_{01} \exp \left[F_{1}(t) x_{1} / k_{B} T\right]
$$




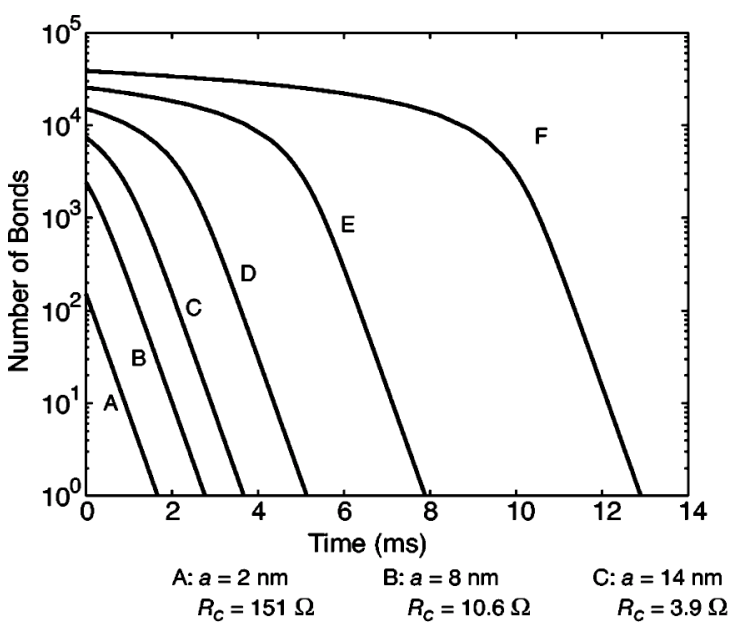

(a)

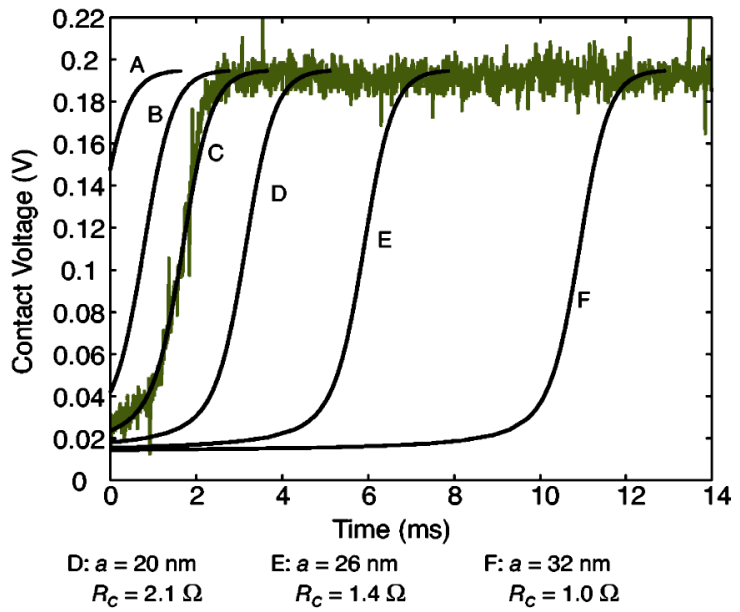

(b)

FIG. 7. (a) Sample solution curves to Eq. (10) with $A_{a}=27.3 \mu \mathrm{m}^{2}$ and (b) corresponding contact voltage plotted with the measured data of Fig. 3. Solutions are shown for various values of the real contact radius $a$ and the corresponding initial contact resistance $R_{c}$ (or resistance before opening begins).

$$
k_{2}(t)=k_{02} \exp \left[F_{2}(t) x_{2} / k_{B} T\right] .
$$

Here, $k_{01}$ and $k_{02}$ are the bond dissociation rates when the force is zero, $k_{B}$ is the Boltzmann constant, and $T$ is the temperature. $x_{1}$ and $x_{2}$ are lengths; the quantities $F_{1} x_{1}$ and $F_{2} x_{2}$ are activation energies, approximately equal to the energy required for bond dissociation. Combining Eqs. (4) and (5) with Eqs. (6) and (7) and considering that $\partial N / \partial t=-N k$ yields, for both bond types,

$$
\begin{aligned}
& \frac{\partial N_{1}}{\partial t}=-N_{1} k_{01} \exp \left[\frac{K_{1} F_{\mathrm{pa}} x_{1}}{k_{B} T\left(K_{1} N_{1}+K_{2} N_{2}\right)}\right], \\
& \frac{\partial N_{2}}{\partial t}=-N_{2} k_{02} \exp \left[\frac{K_{2} F_{\mathrm{pa}} x_{2}}{k_{B} T\left(K_{1} N_{1}+K_{2} N_{2}\right)}\right] .
\end{aligned}
$$

Hence, the result is a pair of coupled differential equations, which may be solved numerically given the initial conditions $N_{10}=\rho_{1} A_{a}$ and $N_{20}=\rho_{2} \pi a^{2}$. Here, $\rho_{1}$ and $\rho_{2}$ are the bond densities for type 1 and type 2 bonds and $A_{a}$ is the apparent contact area (the dimple area).

Equations (8) and (9), and the initial conditions contain eight material parameters: $k_{01}, k_{02}, K_{1}, K_{2}, x_{1}, x_{2}, \rho_{1}$, and $\rho_{2}$. Since the physical makeup of type 1 bonds is not well understood, the four type 1 parameters represent unknowns. However, for gold-gold bonds, the bond density can be estimated as the atomic density for a gold surface, which is $12 \times 10^{6}$ bonds $/ \mu \mathrm{m}^{2}$. The bond stiffness has recently been measured as approximately $12 \mathrm{~N} / \mathrm{m} .{ }^{18}$ Hence, there are still six unknown parameters in the model. These may be found by fitting the model to the measured data.

Given coupled differential equations (8) and (9), the contact opening time $t_{o}$ is the time required for type 2 bonds to reduce to a reasonably small number of bonds. Based on the experimental setup described previously, we define $t_{o}$ as the time required for the contact voltage to reach $97.5 \%$ of the open-circuit voltage. Using the circuit shown in Fig. 2, this corresponds to a contact resistance of about $2.064 \mathrm{k} \Omega$. Using Eq. (1) to calculate the corresponding real contact spot size gives a spot with a radius of about $0.5 \mathrm{~nm}$, or about ten bonds (using the bond density given above). Therefore, the opening time $t_{o}$ was defined as the time required for the number of gold-gold bonds to be reduced to ten bonds.

\section{MODEL PARAMETER EXTRACTION AND DISCUSSIONS}

We used optimization to find the unknown model parameters that fit the data. After optimizing model error from many different starting points, we determined that good fits could only be obtained when $k_{01}$ and $x_{1}$ were set to values which kept the number of type 1 bonds $N_{1}$ nearly constant throughout the time the type 2 bonds dissociated. This implies that the metal bonds dissociate before the contact has mechanically separated. Mechanical measurements using a vibrometer as well as high-frequency electrical measurements are planned to test this hypothesis. However, this finding substantially simplifies the model by eliminating the derivative equation for type 1 bonds. Taking $N_{1}=\rho_{1} A_{a}$, we are left with

$$
\frac{\partial N_{2}}{\partial t}=-N_{2} k_{02} \exp \left[\frac{K_{2} F_{\mathrm{pa}} x_{2}}{k_{B} T\left(K_{1} \rho_{1} A_{a}+K_{2} N_{2}\right)}\right] .
$$

Hence, only three unknown parameters affect the modeling $-k_{02}, x_{2}$, and $K_{1} \rho_{1}$. Two of the original unknowns are combined because they are only found together in Eq. (10).

Sample solutions to Eq. (10) showing gold-gold bonds as a function of time are plotted in Fig. 7(a). Several solutions are shown over a range of real contact radius values, with corresponding contact resistances also indicated. Recall that these real contact radii and contact resistances are defined as the values when the switch is closed, before opening begins. The model solutions can also be converted to predictions of contact voltage as a function of time, allowing a qualitative comparison with the measured contact voltage data shown in Fig. 3. The real contact radius is found as $a(t)=\sqrt{N_{2}(t) / \pi \rho_{2}}$. Equation (1) then allows calculation of the contact resistance $R_{c}$. Lastly, circuit analysis for the testing circuit of Fig. 2 gives the contact voltage $V_{c}$, 


$$
V_{c}=\frac{V_{T}}{50+R_{c}+2 R_{p}}\left(R_{c}+R_{p}\right)
$$

where $V_{T}$ is the total voltage applied by the power supply $(0.2 \mathrm{~V})$ and $R_{c}$ is in Ohms. The probe contact resistance $R_{p}$, in series with $R_{c}$, was taken as $3 \Omega$. Note that the total circuit resistance, in the denominator, includes two probe resistances, while the oscilloscope measures the voltage on just one probe resistance plus the contact resistance. The resulting voltage curves are shown in Fig. 7(b), showing that the model qualitatively matches the data for a contact resistance of $3.9 \Omega$, corresponding to a real contact radius of $14 \mathrm{~nm}$. This value of contact resistance is within the experimental error for the actual contact resistance for the data shown in the figure. Hence, the modeling approach is shown to predict measured trends well.

The three unknown parameters were found by minimizing the prediction error for each of the six main data sets shown in Fig. 4. Since surface chemistry effects are expected to cause some variation for different surfaces, the parameters were found separately for each set. The three parameters were initially found to best fit the data having a contact force $F_{c}$ between 66 and $76 \mu \mathrm{N}$. Then, the value of $K_{1} \rho_{1}$ was found to best fit the data for other force levels, keeping $k_{02}$ and $x_{2}$ equal to their values for the corresponding data at $66-76 \mu \mathrm{N}$. This procedure was used because the nature of the bonds is not expected to change significantly at different contact forces, while the number of bonds may change. The data at $66-76 \mu \mathrm{N}$ was chosen as the baseline data because it represented nearly half of all the data-463 out of 984 points.

The parameters that fit the data are presented in Table II, and the curves fitting the $66-76-\mu \mathrm{N}$ data are shown in Fig. 8 for comparison with the data. The fit quality is very good, particularly considering the large variation in the data with high $A_{a}$. Moreover, despite having been optimized separately, the fitted parameters show reasonably small variability. For all six data sets, the value of $k_{02}$ is $3.76 \pm 0.02 \mathrm{~s}^{-1}$-a $0.5 \%$ spread. The fitted values for $x_{2}$ vary slightly more at $12.65 \pm 2.35 \mathrm{pm}$, a $19 \%$ spread. Using Eq. (5) and the values in Table II, we can calculate the approximate value of $F_{2} x_{2}$ for each switch for the case where $N_{2}$ is ten bonds. The results vary between $3.3 \times 10^{-21}$ and $2.7 \times 10^{-20} \mathrm{~J}$. For comparison, the surface energy of gold in a vacuum is about $1 \mathrm{~J} / \mathrm{m}^{2}$, or about $8.3 \times 10^{-20} \mathrm{~J} /$ bond. Hence, the predicted activation energies are about 3-25 times smaller than the energy per bond in a vacuum. Since these switches are not operating in a perfect vacuum, and impurities are believed to be in the contact area, it is reasonable to expect that the bond energies are smaller than the calculated value. Therefore, the fitted values of $x_{2}$ result in reasonable estimates for the gold bond activation energy.

On the other hand, $K_{1} \rho_{1}$ depends on the apparent contact pressure $\left(F_{c} / A_{a}\right)$, as shown in Fig. 9. This figure plots the fitted values for $K_{1} \rho_{1}$ versus the apparent contact pressure, showing dependence on both the contact force and the apparent area of contact. For apparent contact pressures above about $1 \mathrm{MPa}, K_{1} \rho_{1}$ is fairly constant at about 16000 . However, below $1 \mathrm{MPa}, K_{1} \rho_{1}$ drops sharply and shows much
TABLE II. Model parameters to fit all data sets.

\begin{tabular}{|c|c|c|c|c|c|c|}
\hline Switch & $\begin{array}{c}A_{a} \\
\left(\mu \mathrm{m}^{2}\right)\end{array}$ & $\begin{array}{c}F_{\mathrm{pa}} \\
(\mu \mathrm{N})\end{array}$ & $\begin{array}{c}F_{c} \\
(\mu \mathrm{N})\end{array}$ & $\begin{array}{c}k_{02} \\
(1 / \mathrm{s})\end{array}$ & $\begin{array}{c}x_{2} \\
(\mathrm{pm})\end{array}$ & $\begin{array}{c}K_{1} \rho_{1} \\
\left(\mu \mathrm{N} / \mu \mathrm{m}^{3}\right)\end{array}$ \\
\hline 1 & 26.7 & 76.6 & 75.6 & 3.80 & 12.8 & 16154 \\
\hline \multirow[t]{4}{*}{2} & 27.3 & 76.6 & 14.2 & 3.79 & 12.5 & 16462 \\
\hline & & & 42.0 & 3.79 & 12.5 & 15981 \\
\hline & & & 75.6 & 3.79 & 12.5 & 16225 \\
\hline & & & 116 & 3.79 & 12.5 & 16341 \\
\hline \multirow[t]{4}{*}{3 and 4} & 29.6 & 76.6 & 14.2 & 3.78 & 11.0 & 16143 \\
\hline & & & 42.0 & 3.78 & 11.0 & 16625 \\
\hline & & & 75.6 & 3.78 & 11.0 & 16002 \\
\hline & & & 116 & 3.78 & 11.0 & 16537 \\
\hline \multirow[t]{3}{*}{5} & 29.6 & 54.4 & 35.0 & 3.80 & 10.3 & 16465 \\
\hline & & & 67.2 & 3.80 & 10.3 & 16306 \\
\hline & & & 107 & 3.80 & 10.3 & 15756 \\
\hline \multirow[t]{7}{*}{6} & 169 & 76.6 & 14.2 & 3.76 & 15.0 & 6877 \\
\hline & & & 27.4 & 3.76 & 15.0 & 8036 \\
\hline & & & 42.0 & 3.76 & 15.0 & 7499 \\
\hline & & & 75.6 & 3.76 & 15.0 & 9517 \\
\hline & & & 116 & 3.76 & 15.0 & 9772 \\
\hline & & & 168 & 3.76 & 15.0 & 12281 \\
\hline & & & 236 & 3.76 & 15.0 & 11250 \\
\hline \multirow[t]{5}{*}{7} & 314 & 76.6 & -9.0 & 3.76 & 15.0 & 5243 \\
\hline & & & 14.2 & 3.76 & 15.0 & 7055 \\
\hline & & & 75.6 & 3.76 & 15.0 & 12057 \\
\hline & & & 116 & 3.76 & 15.0 & 13167 \\
\hline & & & 236 & 3.76 & 15.0 & 11210 \\
\hline
\end{tabular}

more variability, suggesting that low-pressure contacts form fewer type 1 bonds with more variability in the bonds. The modeling performed here cannot separate the effects of changing bond density and bond stiffness. However, it is reasonable to assume that larger apparent pressures cause the surfaces to come into closer proximity. This could allow more type 1 bonds to form; alternatively, if type 1 bonds represent predominately van der Waals interactions, we would expect to see increased bond stiffness, since van der Waals forces vary with a high power of surface separation. This variation in $K_{1} \rho_{1}$ also explains why the contacts with large apparent contact area show more variability in the opening time. These large-dimple contacts had small apparent contact pressure, resulting in considerable variation in the formation of type 1 bonds.

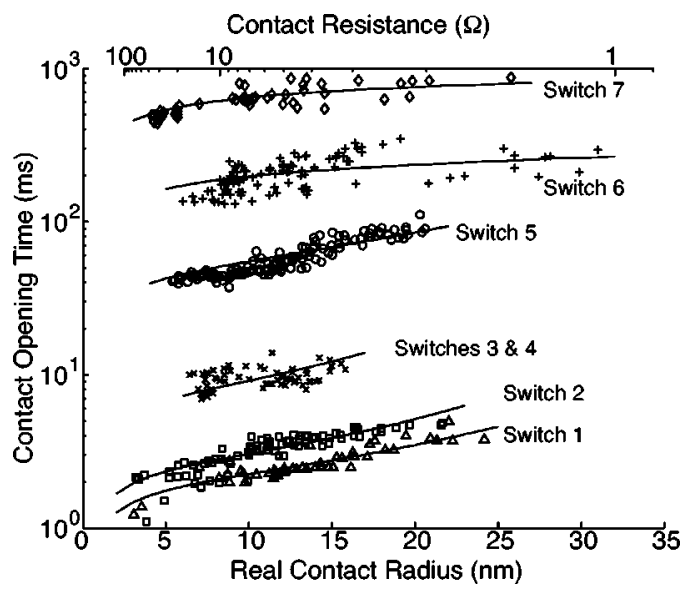

FIG. 8. Comparison of the model to the data at a contact force of $66-76 \mu \mathrm{N}$. 


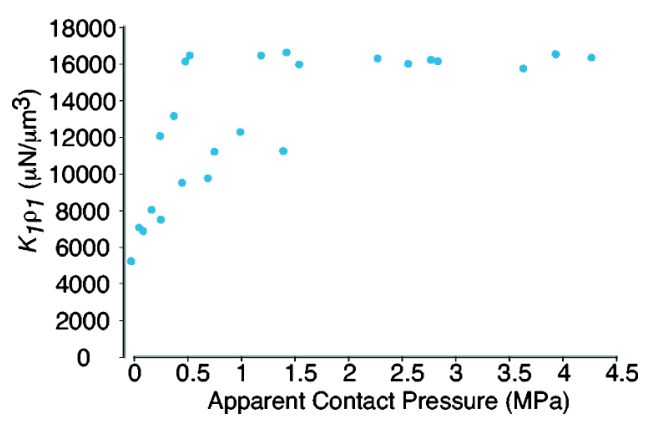

FIG. 9. $K_{1} \rho_{1}$ as a function of the apparent contact pressure for all data.

\section{HIGH CONTACT FORCE OPERATION FOR FAST SWITCHING}

One other issue remains to be highlighted: the contact opening times shown in Fig. 4 are too long. Even the fastest measurements are still above $1 \mathrm{~ms}$, while $10-50 \mu \mathrm{s}$ is desired. ${ }^{1}$ However, we have found that the contact opening time is drastically reduced for contacts with small apparent contact area and large contact force. The switches with an apparent contact area of $29.6 \mu \mathrm{m}^{2}$ or smaller showed significantly smaller contact opening times when the contact force was high. This is illustrated in Fig. 10. This figure shows the contact opening time for switches 2 and 3, with $A_{a}$ of 27.3 and $29.6 \mu \mathrm{m}^{2}$ as a function of actuation force. The symbols on the left of the plot, representing contact force less than $158 \mu \mathrm{N}$, show the data already presented in Fig. 4. As the contact force increases beyond this point, the contact opening time decreases. In fact, at sufficient contact force, the opening times are approximately 100-1000 times smaller than at low force, and all of the opening times line up at one of four levels-5.3, 8.1, 11.5, or $13.9 \mu$ s (suggesting that the very fast opening times are quantized).

Moreover, the figure shows that for switch 2 with $A_{a}$ $=27.3 \mu \mathrm{m}^{2}$, contact opening time begins to decrease when the contact force rises above about $160 \mu \mathrm{N}$. By the time $F_{c}$ has reached $236 \mu \mathrm{N}$, the opening times line up at one of the four levels noted above. The data for switch 3 with $A_{a}$ $=29.6 \mu \mathrm{m}^{2}$ show the same behavior, though the large drop in

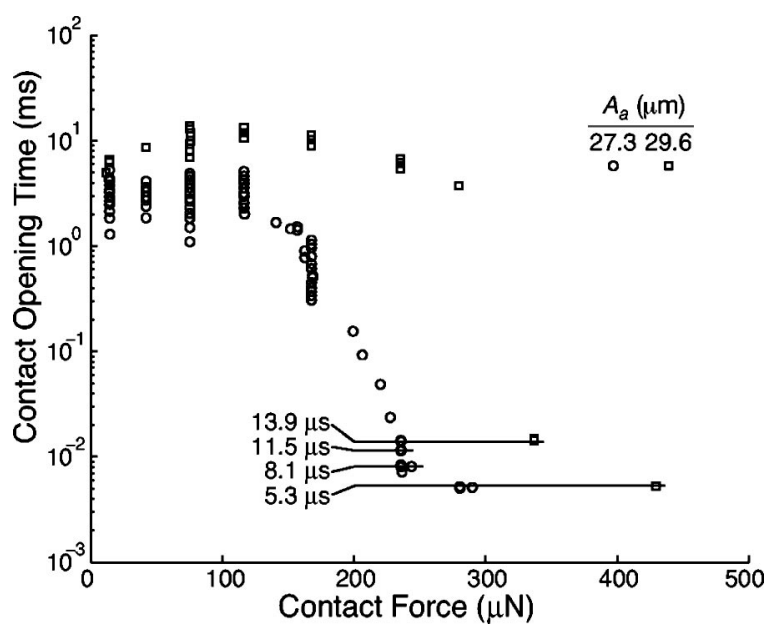

FIG. 10. High-force data for $A_{a}$ of 27.3 and $29.6 \mu \mathrm{m}^{2}$, showing contact opening time vs contact force.

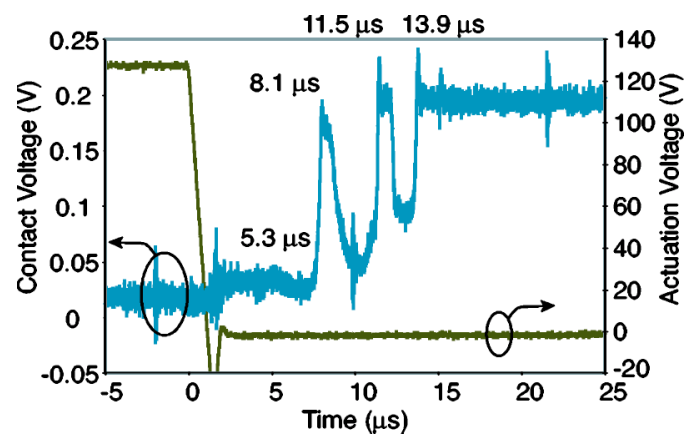

FIG. 11. (Color online) Oscilloscope trace of a contact voltage showing dynamic effects.

opening time is not seen until $F_{c}$ is $336 \mu \mathrm{N}-\mathrm{a}$ higher force than was required by the switch with the smaller apparent contact area.

The fact that the very fast contact opening times are quantized indicates that a different mechanism is controlling the contact opening-switch vibrations. The model data of Fig. 8 were made without considering dynamic loads due to vibrations. Essentially, the contacts required enough time to open that the mechanical vibrations had damped considerably by the time the contacts began to open. However, a sample oscilloscope trace for a fast contact opening time, shown in Fig. 11, illustrates behavior very different than the slower opening shown in Fig. 3. The traces in Fig. 11 were taken on switch 2 at an actuation voltage of $128.1 \mathrm{~V}$, corresponding to a contact force of $236 \mu \mathrm{N}$. Rather than the relatively smooth increase of Fig. 3, the contact voltage of Fig. 11 has peaks at $13.9,11.5$, and $8.1 \mu$ s, with a smaller peak at $5.3 \mu \mathrm{s}$. These peaks correspond exactly with the contact opening time levels described in Fig. 10. Moreover, all the measurements made for small dimple sizes at high force showed the same behavior, with peaks occurring at the same times to within about $0.5 \mu \mathrm{s}$. We believe this effect is related to mechanical vibrations of the switch.

To further explore the dynamic properties of the switch, we calculated the resonant period for one-half of the switch, assuming that the dimple at the center of the switch is adhered to the substrate. This is done by solving the fourthorder beam deflection equation,

$$
E I \frac{\partial^{4} w}{\partial x^{4}}-F_{a} \frac{\partial^{2} w}{\partial x^{2}}=-\rho_{g} A \frac{\partial^{2} w}{\partial t^{2}} .
$$

Here, $E I$ is the beam's flexural rigidity, $w$ is the transverse deflection of the beam, $x$ is the coordinate along the beam's length, $F_{a}$ is the axial force (assumed tensile) acting on the beam due to residual stress, $\rho_{g}$ is the density of the beam, and $A$ is the cross-sectional area of the beam. The solution to this equation using the method of separation of variables leads to an expression describing mode shapes and fundamental frequencies of the beam. The solution technique is described in many texts; see Ref. 19 for an example. By comparing beam deflections to a mechanical model, Young's modulus $E$ and axial force $F_{a}$ were measured as $43.5 \mathrm{GPa}$ and $8.6 \mathrm{mN}$, respectively. The density of the sputtered gold is approximated as $18 \mathrm{pg} / \mu \mathrm{m}^{3}$, as reported in Ref. 20. Using these values, we calculate the period of fundamental resonance as about 


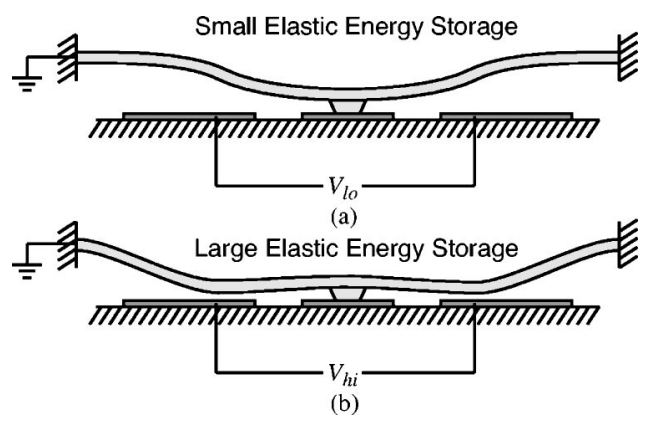

FIG. 12. An illustration of the beam deflection (a) at low contact force and (b) at high contact force. Significantly more elastic energy is stored in the beam for case (b).

$3.6 \mu \mathrm{s}$, with a second-mode resonant period of $1.4 \mu \mathrm{s}$. For comparison, the period between the peaks in Fig. 11 is approximately $3 \mu \mathrm{s}$. The reasonable agreement between the experiments and the calculated value suggests that mechanical vibrations are responsible for the observed behavior.

Therefore, we conclude that the fast-opening contacts are governed by switch vibrations. As actuation voltage rises, more elastic energy is stored in the switch in the down state, as suggested in Fig. 12. This figure illustrates the beam deflection for low contact force in part (a) and high contact force in part (b). The larger deflections throughout the beam for the high-force case mean that significantly more elastic energy is stored in the beam. When this energy is released by turning off the actuation voltage, it causes mechanical vibrations in the switch beam, introducing transient pull-apart forces higher than the equilibrium pull-apart forces shown in Table II. For large dimples and low actuation voltages, the transient pull-apart forces are not high enough to cause release of the contact, and they may be ignored. However, Fig. 10 suggests that the smaller dimples tested here can exhibit dynamic effects at sufficient contact force. We believe that the quantized levels for contact opening time represent maxima in the transient pull-apart force. As the switch vibrates when the actuation voltage is turned off, the contact releases at one of these high points in the transient pull-off force. Note also that recent results by Kogut and Komvopoulos have also demonstrated reduced adhesion under larger actuation voltage for micromechanical components. ${ }^{21}$

Returning to the data in Fig. 10, recall that the transition to the fast opening times occurred at a lower contact force for switch 2 than it did for switch 3. Apparently, since the larger apparent contact area in switch 3 causes more adhesion, the transition to vibrational behavior occurs at a higher force. Therefore, reduction in the dimple area reduces the force required for the transition to dynamic effects, further encouraging the design of small apparent contacts. More work is being done to model this behavior.

\section{CONCLUSIONS}

This work has demonstrated measurements which characterize adhesion effects in micromachined switches. The method measures the time required for the switch contact to open. Micromechanism adhesion has traditionally been characterized by observing quasistatic sticking of surfaces. The technique demonstrated here directly measures the effect adhesion has on switching speed, providing information directly applicable to switch operation. Moreover, the measurement is nondestructive, allowing multiple measurements to be made on a single switch.

Using this technique, we performed 1088 tests of switch opening time. The results show that switch opening time increases when the apparent contact area is larger, or if the contact resistance or pull-apart force is smaller. We have linked the first trend with adhesive forces acting on the apparent contact area, regardless of the size of the real contact spot. We believe that the second is caused by increased adhesion in contacts with a larger real metal-to-metal contact area. The third trend (faster opening when contact opening force is larger) is certainly not surprising, but the data given here allows its effect to be modeled.

Accordingly, we have also developed a model to explain the experimental observations. We used the data to extract model parameters. In this way, we showed that the extracted values of bond activation energy compare well with published data on gold bond energy. We also found that the bond density or bond stiffness for type 1 bonds (those not at the real contact spot) increases with apparent contact pressure until a maximum is reached at approximately $1 \mathrm{MPa}$. This suggests that these bonds depend on the proximity of the contacting surfaces, with greater proximity resulting in either more bonds or stiffer bonds.

Finally, we showed that contacts created with small apparent contact area and large contact force demonstrated significantly reduced contact opening time. We suggested that this is due to mechanical vibrations which lead to large transient pull-apart forces. Analysis of the extremely fastopening switches showed that opening times were quantized with a period nearly the same as the calculated resonant period for beam vibrations. Moreover, we found that switches with smaller apparent contact area made the transition to faster opening times at lower contact force.

The model and data suggest recommendations for switch design. In order to increase switching speed, the restoring force should be as large as possible while the dimple size must be as small as possible. Increasing contact resistance also improves switching speed, though at the expense of switch performance. Hence, the dimple size and pull-apart force must be designed to allow fast pull off even when the contact resistance is low. Since a large switch stiffness normally makes a high actuation voltage necessary, this represents a design trade-off, with the stiffness of the switch chosen to give a reasonably small actuation voltage while still maintaining a sufficient pull-apart force for contact opening. However, no such trade-off exists for dimple size. Since contact resistance shows no dependence on apparent contact area, ${ }^{22}$ fabrication limitations should be the only factors restricting the size of the apparent contact. Therefore, a strong recommendation of this work is that contact dimples be made as small as possible to reduce switching time. Moreover, small contact dimples also enable the transition to vibrational effects, significantly reducing opening time. 


\section{ACKNOWLEDGMENTS}

This work was supported by the National Science Foundation under Contract Nos. ECS-0115222 and ECS-0330963 and by the Central Intelligence Agency under Contract No. 2001-H605400-000. One of the authors (B.D.J.) appreciates the support of the Department of Defense through a National Defense Science and Engineering Graduate Fellowship. One of the authors (K.K.) thanks the National Science Foundation for its support through a CAREER award.

${ }^{1}$ G. M. Rebeiz and J. B. Muldavin, IEEE Microw. Mag. 2, 59 (2001).

${ }^{2}$ C. H. Mastrangelo and C. H. Hsu, J. Microelectromech. Syst. 2, 33 (1993).

${ }^{3}$ C. H. Mastrangelo and C. H. Hsu, J. Microelectromech. Syst. 2, 44 (1993).

${ }^{4}$ M. P. de Boer and T. A. Michalske, J. Appl. Phys. 86, 817 (1999).

${ }^{5}$ W. M. van Spengen, R. Puers, and I. De Wolf, J. Micromech. Microeng. 12, 702 (2002).

${ }^{6}$ P. Decuzzi and D. J. Srolovitz, J. Microelectromech. Syst. 13, 377 (2004).

${ }^{7}$ C.-C. Lee and W. Hsu, J. Vac. Sci. Technol. B 21, 1505 (2003).

${ }^{8}$ B. D. Jensen, L.-W. Chow, K. Huang, K. Saitou, J. L. Volakis, and K.
Kurabayashi, J. Microelectromech. Syst. (to be published).

${ }^{9}$ J. W. Tringe, T. A. Uhlman, A. C. Oliver, and J. E. Houston, J. Appl. Phys. 93, 4661 (2003).

${ }^{10}$ B. D. Jensen, Ph.D. thesis, University of Michigan, 2004.

${ }^{11}$ R. Holm, Electric Contacts-Theory and Applications, 4th ed. (Springer, Berlin, 1967).

${ }^{12}$ B. Nikolić and P. B. Allen, Phys. Rev. B 60, 3963 (1999).

${ }^{13}$ W. Yan and K. Komvopoulos, J. Appl. Phys. 84, 3617 (1998).

${ }^{14}$ N. W. Ashcroft, N. D. Mermin, and D. Mermin, Solid State Physics, 1st ed. (Holt, Rinehart and Winston, New York, 1976).

${ }^{15}$ U. Seifert, Phys. Rev. Lett. 84, 2750 (2000).

${ }^{16}$ G. I. Bell, Science 200, 618 (1978).

${ }^{17}$ K. Prechtel, A. R. Bausch, V. Marchi-Artzner, M. Kantlehner, H. Kessler, and R. Merkel, Phys. Rev. Lett. 89, 28101/1 (2002).

${ }^{18}$ G. Rubio-Bollinger, S. R. Bahn, N. Agraï, K. W. Jacobsen, and S. Vieira, Phys. Rev. Lett. 87, 026101 (2001).

${ }^{19}$ S. S. Rao, Mechanical Vibrations, 2nd ed. (Addison-Wesley, Reading, MA, 1990)

${ }^{20}$ D. Lützenkirchen-Hecht and R. Frahm, Physica B 283, 108 (2000).

${ }^{21}$ L. Kogut and K. Komvopoulos, Appl. Phys. Lett. 84, 4842 (2004).

${ }^{22} \mathrm{~S}$. C. Bromley and B. J. Nelson, in Proceedings of the 47th IEEE Holm Conference Electrical Contacts, Montreal, Quebec, Canada, 10-12 Sept. 2001 (IEEE, Piscataway, NJ, 2001), pp. 122-127. 